Résumés des conférences et travaux

\title{
Philologie grecque
}

Brigitte Mondrain

\section{OpenEdition \\ Journals}

Édition électronique

URL : https://journals.openedition.org/ashp/1074

DOI : $10.4000 /$ ashp. 1074

ISSN : 1969-6310

Éditeur

Publications de l'École Pratique des Hautes Études

\section{Édition imprimée}

Date de publication : 2 février 2011

Pagination : 375-378

ISSN : 0766-0677

\section{Référence électronique}

Brigitte Mondrain, "Philologie grecque », Annuaire de l'École pratique des hautes études (EPHE), Section des sciences historiques et philologiques [En ligne], 141 | 2011, mis en ligne le 16 mars 2011, consulté le 06 juillet 2021. URL : http://journals.openedition.org/ashp/1074 ; DOI : https://doi.org/10.4000/ashp. 1074 


\section{PHILOLOGIE GRECQUE*}

\section{Directeur d'études : M ${ }^{\mathrm{me}}$ Brigitte Mondrain}

Programme de l'année 2008-2009: I. Histoire des livres, histoire des textes et histoire de la culture : étude de manuscrits scientifiques, historiques et théologiques. - II. Questions de paléographie et de codicologie.

\section{Histoire des livres, histoire des textes et histoire de la culture :} étude de manuscrits scientifiques, historiques et théologiques

On est parti cette année de l'étude d'un livre qui non seulement fut novateur en son époque mais demeure un ouvrage de référence aujourd'hui : la Palaeographia Graeca, parue à Paris en 1708 et due à Bernard de Montfaucon, moine bénédictin comme Jean Mabillon et contemporain de lui. Cet ouvrage crée un nouveau terme, celui de «paléographie », mais il marque surtout les débuts d'une nouvelle discipline, définie et construite avec un grand brio par son auteur. La Griechische Palaeographie de Victor Gardthausen, qui fut publiée en 1879 puis en 1911-1913 dans une nouvelle édition en deux volumes et qui est à l'heure actuelle encore la synthèse la plus riche pour le domaine de l'écriture grecque byzantine, sinon la plus complète au regard des progrès effectués par la discipline, n'a pas rendu l'œuvre de Montfaucon obsolète.

Le titre complet de ce volume in-folio d'un peu plus de 600 pages est Palaeographia Graeca, sive de ortu et progressu literarum Graecarum, « de l'origine et du développement des lettres grecques », notation qui est suivie, en plus petits caractères sur la page, de ces précisions : et de variis omnium saeculorum Scriptionis Graecae generibus : itemque de Abbreviationibus et de Notis variarum Artium ac Disciplinarum, « et des différentes sortes d'écritures grecques au cours des siècles; également des abréviations et des notations propres à divers arts et disciplines ».

Dans le premier livre de l'ouvrage, les chapitres 6 et 7 dressent une liste détaillée de manuscrits, en particulier des manuscrits datés, qui offrent le nom du copiste noté dans une souscription, éditée en grec et traduite en latin. Il s'agit de livres que Montfaucon connaît pour les avoir lui-même examinés dans les bibliothèques de diverses villes d'Europe, en particulier italiennes, et de divers collectionneurs (telle la collection de Colbert pas encore incorporée à la bibliothèque du roi de France). Certains de ces manuscrits se retrouvent cités et utilisés plus loin dans la Palaeographia Graeca pour éclairer un développement précis sur des caractéristiques de l'écriture. L'on a choisi de s'arrêter plus particulièrement sur un scribe nommé Athanase, qui a signé l'achèvement de son travail dans un manuscrit dont la cote était alors Codex Regius 2385 (Parisinus gr. 857 aujourd'hui). Comme le précise Montfaucon à la p. 65, en donnant la transcription intégrale de la souscription et sa traduction latine, Athanase a écrit à l'aide de monocondyles. Si bien que ce colophon est à nouveau cité et étudié

* L'arrivée tardive de ce résumé n'a pas permis de l'insérer à sa place dans cet Annuaire. 
dans le livre $\mathrm{V}$, consacré aux différentes manières d'écrire de manière abrégée ou liée, « De abbreviationibus ac de notis disciplinarum et artium » aux p. 349-350.

Le cas montre en effet la qualité du travail novateur réalisé par Montfaucon pour réunir et analyser des données, acquises grâce à une étude directe des livres; en même temps, il témoigne de l'influence éventuellement négative qu'a suscitée au fil des siècles le respect légitime dû à son remarquable ouvrage : des incertitudes voire des erreurs ont pu se glisser dans la grande quantité d'informations rassemblées par lui et le lecteur se doit de garder l'esprit critique en éveil. Le cas de cet Athanase permet d'illustrer les modalités de la lecture des textes et constitue en quelque sorte un exemple pour la critique textuelle, en mettant en évidence la manière dont des erreurs peuvent, d'auteur à auteur, se succéder, être répétées voire amplifiées au fil du temps.

La souscription du moine Athanase est donc écrite en monocondyles, ces jeux d'écriture qui consistent à lier, sans relever le calame et à l'aide de souples volutes, plusieurs lettres à la suite : «per Monocondylia, sive per longos et perplexos literarum nexus ». La lecture en est souvent délicate car les caractères tendent à être déformés dans ces tracés où priment les arrondis. Le Parisinus gr. 857 présente une telle souscription écrite sur six lignes, ce qui est assez rare. La transcription, déjà donnée à la p. 65, est reprise à la p. 349 de la Palaeographia Graeca et précède là une illustration de ce colophon, annoncée en deuxième place sur une page de planches consacrée aux monocondyles ( « Secunda Monocondiliorum Tabula ») ; la page 350 offre deux fac-similés dessinés mais notre souscription se trouve en réalité en premier lieu et non en second comme l'annonçait Montfaucon (cette interversion dans la mise en page a suscité une erreur, vénielle, pour le choix de la transcription donnée en regard de la reproduction dans un récent petit manuel de paléographie grecque en ligne à l'usage des étudiants). La présence de quelques illustrations, de belle qualité, est en effet une des caractéristiques de l'ouvrage de Bernard de Montfaucon.

Celle-ci a connu un certain succès puisqu'elle se trouve reproduite pour illustrer le phénomène des monocondyles dans les deux éditions successives de la Griechische Palaeographie de Victor Gardthausen, à la p. 114 de l'édition de 1879 et p. 51 de celle de 1913. La lecture que Gardthausen en propose est celle de Montfaucon : elle est partiellement fautive, pour ce qui est de la localisation de la copie dans un monastère « tou génèsiou » et non « tou galèsiou ». Mais il vaut aussi la peine de noter que cette mélecture du nom du monastère avait été corrigée dès 1888 par Henri Omont qui, à la différence de Gardthausen, avait examiné le manuscrit directement : dans la Revue critique, N. S. t. XXVI de 1888 (où il signe $\Omega$ ), il offre une recension brève de l'ouvrage de John W. Bradley, A Dictionary of Miniaturists, Illuminators, Calligraphers, and Copyists, paru à Londres en 1887; il donne quelques additions et corrections pour la lettre A du dictionnaire, en ce qui concerne les copistes de manuscrits grecs de la Bibliothèque nationale de Paris, étant donné qu'il réalisait alors son catalogue sommaire de ce fonds. Et en 1891, dans son livre Fac-similés des manuscrits grecs datés de la Bibliothèque nationale du IXe au XIV siècle, il transcrit correctement (p. 12) le nom de ce monastère du Galesion, qui se trouve près d'Ephèse. En 1909, Maria Vogel et Victor Gardthausen dans leur répertoire de copistes grecs reprennent à juste titre cette attribution, en renvoyant aux Fac-similés d'Omont. Et pourtant Gardthausen choisit de ne pas la retenir et de revenir à «Génèsiou » dans la seconde édition de sa 
Griechische Palaeographie, p. 51 et 481 (où il récuse même dans une note la lecture de «Galèsiou » adoptée par le livre réalisé avec M. Vogel). L'examen attentif du dessin dans l'ouvrage de Montfaucon permet d'expliquer cette persévérance de Gardthausen dans l'erreur. De fait, la même souscription offerte sous forme de photographie dans le recueil Les manuscrits grecs datés des XIII et XIVe siècles dans les bibliothèques publiques de France. I. XIII ${ }^{e}$ siècle, paru en 1989, présente un tracé plus clair après le gamma initial du terme; on a bien une ligature d'alpha et lambda et non d'epsilon et $n u$. Il faut donc souligner que les gravures dans le livre de Montfaucon ont été faites sur la base des dessins soigneusement réalisés par l'auteur mais tributaires de la lecture qu'il effectuait lui-même, ce qui a contribué à déformer les tracés réels !

Bernard de Montfaucon situe ce manuscrit Parisinus gr. 857, copié sur parchemin, au XIII ${ }^{\mathrm{e}}$ siècle mais ne le date pas précisément. La souscription donne en effet le jour du mois, 17 février, le jour de la semaine, un jeudi, et la mention de l'indiction, 4 e indiction, mais pas la mention de l'année. C'est Gardthausen qui a résolu en 1879 la devinette en notant que la $4^{\mathrm{e}}$ indiction, au cours du XIII ${ }^{\mathrm{e}}$ siècle, tombe un jeudi 17 février seulement en 1261.

Mais Gardthausen reproduit pour le reste en l'interprétant et la développant la notice de Montfaucon située à la p. 65 de la Palaeographia Graeca. Dans cet ouvrage, le copiste Athanase du Parisinus gr. 857 est rapproché (« ejusdem, ut videtur, Athanasii ») d'un scribe du même nom responsable selon Montfaucon de deux manuscrits, qui sont copiés sur papier oriental : un manuscrit achevé le samedi 8 juillet 1273, le Regius 2753 (Parisinus gr. 2654) de l'Etymologicum magnum, et explicitement signé par un moine Athanase et un autre, le voisin sur les étagères de la Bibliothèque royale du temps de Montfaucon, le Regius 2754 (Parisinus gr. 2408), au contenu composite; le second n'est pas signé mais l'écriture est évidemment la même d'après Montfaucon : «ut ex Charactere liquidum ». Le rapprochement de ces trois manuscrits, dont deux sont assurément dus à un moine Athanase, ont été le point de départ d'attributions fausses et maintes fois répétées jusqu'à la fin du XIX ${ }^{\mathrm{e}}$ siècle et au-delà. Cela d'autant plus que Victor Gardthausen a repris l'erreur en l'amplifiant. Tout d'abord en attribuant à un même copiste Athanase le Parisinus gr. 2654, explicitement daté de 1273, et le Parisinus gr. 2408, arbitrairement daté par lui de 1270; la raison de cette datation arbitraire est à chercher dans le Catalogus codicum Manuscriptorum Bibliothecae Regiae Parisiensis de 1740, p. 496-497 qui, en se fondant sans aucun doute sur l'identification proposée par Montfaucon, présente le codex 2408 ainsi : " is codex manu Athanasii Hamartoli circa annum Christi 1270 exaratus est » (puisque le Parisinus gr. 2654 est, lui, de 1273). Cette datation de 1270 sera reprise par Bradley, qui se trompe dans la cote du manuscrit « $2508 »$. Henri Omont corrige l'erreur de cote mais complète la faute de datation et d'attribution à une même main dans la Revue critique de 1888 : «Athanase, moine, copiste en 1273 (et non 1270) du ms. Paris. gr. 2654 et des fol. 142-165 du ms. 2408 (et non 2508) » (on retrouve la même affirmation dans l'Introduction à l' Inventaire sommaire); une nouvelle étape est alors franchie, étant donné que ces deux manuscrits ne présentent absolument pas la même main. Omont distingue néanmoins deux Athanase, celui du Parisinus gr. 857 et un autre intervenant dans deux codices différents. 
Gardthausen va encore plus loin, en attribuant à un même copiste Athanase non seulement ces trois manuscrits mais également deux autres : l'un de Munich, simplement parce qu'il a été copié au XIII ${ }^{\mathrm{e}}$ siècle, selon le catalogue, par un copiste Athanase, le Monacensis gr. 201 ; l'autre par suite d'une erreur manifeste dans ses fiches qui l'a amené dès 1879 à considérer que la longue souscription en monocondyles se trouvait non pas dans le Parisinus gr. 857 mais dans le Parisinus gr. 2992, ensuite noté 2292 (p. 403). La confusion de cote est discrètement corrigée dans l'édition de 1913 mais seulement partiellement (p. 481), si bien que le Parisinus gr. 2292, qui est un manuscrit de Paul d'Égine (d'où la confusion avec Paul de l'Évergétis que contient le Parisinus gr. 857 ?) et dans lequel aucun Athanase n'est mentionné, est toujours cité comme témoin dû à notre Athanase :

wenn aber der Par. 2292, der durch seine Monokondylien ohne Jahreszahl merkwürdig ist, vom Athanasius 1261 geschrieben wurde, so muss auch der c. Monac. 201 (s. XIII) ungefähr gleich alt sein, weil derselbe ebenfalls von Athanasius geschrieben ist...

Nigel Wilson dans un bref article paru dans Scriptorium en 1961, avait déjà souligné que l'on avait affaire à des Athanase différents, si tant est qu'il s'agisse d'Athanase. On a ici, tout en examinant ces divers manuscrits, reconstitué les méandres qui risquent de conduire, d'interprétation extrapolée en affirmation trop rapide, à des fautes reproduites sans vérification préalable des données.

On s'est ensuite intéressé plus précisément au Parisinus gr. 857, à son écriture et à l'activité de copie que l'on peut reconstituer au monastère du Galésion dans la seconde moitié du XIII ${ }^{\mathrm{e}}$ siècle, sur la base de souscriptions, de marques de possession du monastère et de l'examen d'un même type d'écriture que l'on retrouve dans plusieurs manuscrits qui ont été successivement examinés. Le Parisinus gr. 857, qui contient le livre IV de la Collection ascétique en quatre livres de Paul de l'Évergétis, est lui-même particulièrement intéressant en ce sens car, outre sa souscription en monocondyles, il offre une longue note d'une centaine de dodécasyllabes; rédigée par Athanase, elle précise entre autres les conditions d'élaboration du livre et permet de le situer dans un contexte historique. D'ailleurs, la main même d'Athanase, erronément repérée dans divers manuscrits, se trouve en fait aussi sur deux pages d'un manuscrit de Ménées de novembre, écrit en 1259-1260 et dû sinon à un moine Barlaam, le Vaticanus Reginensis gr. 63, comme l'avait justement supposé Alexander Turyn.

\section{Questions de paléographie et de codicologie}

Dans le cadre du séminaire d'introduction à l'étude des manuscrits byzantins, on a lu et analysé sur le plan de la codicologie, de l'histoire des textes et de l'histoire des livres plusieurs manuscrits significatifs par leur écriture. 